\title{
Open total arch replacement with trifurcated graft and frozen elephant trunk
}

\author{
Erik Beckmann", Andreas Martens", Wilhelm Korte, Tim Kaufeld, Heike Krueger, Axel Haverich, \\ Malakh Shrestha
}

Department of Cardio-Thoracic, Transplantation and Vascular Surgery, Hannover Medical School, Hannover, Germany

\#These authors contributed equally to this work.

Correspondence to: Malakh Shrestha, MD. Department of Cardiothoracic, Transplantation and Vascular Surgery, Hannover Medical School, Carl-

Neuberg-Strasse 1, 30625 Hannover, Germany. Email: shrestha.malakh.lal@mh-hannover.de.

\begin{abstract}
Background: The frozen elephant trunk (FET) technique was introduced in Hannover Medical School in 2001 to treat patients with complex aortic arch pathologies. Since 2012, we primarily use the trifurcated Thoraflex Hybrid FET graft. In this article, we report our experience with the trifurcated FET graft.

Methods: Between November 2012 and September 2018, 211 patients underwent FET implantation with the trifurcated Vascutek Thoraflex Hybrid graft. The indications for surgery were: degenerative aneurysms in 68 patients, acute aortic dissections (AD) in 96 patients, and chronic ADs in 47 patients. And, 18\% of cases were sternal re-operations.

Results: Mean cardiopulmonary bypass time, aortic cross-clamp time, and myocardial ischemia time were $262 \pm 84,115 \pm 71,50 \pm 26$ minutes, respectively. Incidence of re-thoracotomy for bleeding, stroke, permanent paraplegia/paraparesis, prolonged ventilatory support (>96 h), and long-term dialysis were 13\%, 18\%, 2\%, $21 \%$, and $5 \%$, respectively. In-hospital mortality was $12 \%$. Follow-up was complete for $100 \%$ of patients and comprised a total of 513 patient years. The mean follow-up time was 2.2 [0-6] years. During follow-up, there were 32 aortic re-interventions distal to the FET. The survival rate at 1 and 5 years was $84 \%$ and $81 \%$, respectively.

Conclusions: Total aortic arch replacements with trifurcated FET can be performed with positive results. The trifurcated graft allows selective anastomosis of the supra-aortic vessels, which might result in improved hemostasis.
\end{abstract}

Keywords: Aortic arch surgery; frozen elephant trunk (FET); trifurcated graft; zone 2 anastomosis

Submitted Nov 25, 2019. Accepted for publication Mar 03, 2020.

doi: $10.21037 /$ acs.2020.03.09

View this article at: http://dx.doi.org/10.21037/acs.2020.03.09

\section{Introduction}

Total aortic arch replacement has been and remains, a complex operation. However, even after successful operations, many patients require further repair of the distal aorta. Open surgical repair of the descending aorta represents a difficult operation, especially when the proximal anastomosis needs to be connected to a previously implanted aortic arch graft. The introduction of the "elephant trunk" (ET) simplified this operation tremendously (1).

The "frozen elephant trunk" (FET) technique was developed by Karck and colleagues (1), who modified Borst's ET technique by using a stent graft to secure the distal ET section (2). This allows treatment of complex pathologies of the aortic arch and proximal descending aorta in a single-step procedure, and facilitates second-stage repair, if needed (3).

In 2012, working in close collaboration with Vascutek Terumo, we introduced the Thoraflex hybrid FET graft (4). This novel composite prosthesis combines a trifurcatedbranched gelatin-coated woven polyester graft and a distal stented graft with a simple deployment mechanism. The 


\begin{tabular}{|c|c|c|c|c|c|}
\hline Number of patients & 211 (100\%) & $68(32 \%)$ & $96(46 \%)$ & 47 (22\%) & \\
\hline Age (years) & $59.2 \pm 12.5$ & $66.9 \pm 9.8$ & $56.2 \pm 11.2$ & $54.4 \pm 13.7$ & $<0.001$ \\
\hline Sex, male & $139(66 \%)$ & $33(49 \%)$ & $72(75 \%)$ & $34(72 \%)$ & 0.001 \\
\hline Sternal re-operation & 37 (18\%) & $5(7 \%)$ & $1(1 \%)$ & 31 (66\%) & $<0.001$ \\
\hline Diagnosis & & & & & $<0.001$ \\
\hline Aneurysm & $68(32 \%)$ & $68(100 \%)$ & $0(0 \%)$ & $0(0 \%)$ & \\
\hline Acute dissection & $96(46 \%)$ & $0(0 \%)$ & 96 (100\%) & $0(0 \%)$ & \\
\hline
\end{tabular}

Acute malperfusion refers to acute ischemic syndromes in acute dissection patients. AD, acute dissection; CD, chronic dissection; An, degenerative aneurysm; BMI, body mass index.

woven polyester graft has three branches for aortic arch connection, and one extra branch for perfusion. The Thoraflex hybrid is the only commercially available FET prosthesis with side branches in Europe and the United States of America.

Despite surgical and technological advances, total aortic arch replacement with a FET graft remains a challenging procedure, which requires a period of hypothermic circulatory arrest (HCA). Peri-operative mortality and morbidity has improved substantially over the recent years, but still ranges from 4-17\% (5-10).

In a previous report, we described our experience of the first 100 operations using the Thoraflex graft (7). In this manuscript, we report our most recent experience with the trifurcated FET graft.

\section{Methods}

\section{Patients}

Between November 2012 and September 2018, 211 patients underwent total aortic arch replacement with the Vascutek Thoraflex hybrid prosthesis. We analyzed the data according to the underlying disease (degenerative aneurysm (An), N=68; acute aortic dissection (AD) (Stanford type A, DeBakey I, N=96) and chronic aortic dissection (CD) $(\mathrm{N}=47)$. This retrospective study was approved by the institutional ethics committee.

Patients were followed either through regular visits at our outpatient clinic or actively contacted by a study nurse team. Clinical data was collected from referring physicians after informed consent. Follow-up ended in December 2017 and was $100 \%$ complete.

Pre-operative patient characteristics are shown in Table 1.

\section{Peri-operative management}

The cerebrospinal fluid (CSF) is drained such that the spinal pressure remains below ca. $12 \mathrm{mmHg}$. The CSF drainage catheter is inserted just prior to the operation in elective operations or, in acute cases, immediately after the operation in the intensive care unit. Patients with coagulopathies receive CSF drainage only after normalizing the international normalized ratio (INR) to a maximum of 1.2.

\section{Surgical technique}

After a median sternotomy, extra-corporeal circulation (ECC) is initiated with direct cannulation of the aorta and the right atrium. Direct aortic cannulation using the Seldinger technique under transesophageal echocardiography (TEE) guidance is our standard method for arterial access, including AD cases. Axillary or femoral cannulation is only used in selected patients. The left heart is vented through the right superior pulmonary vein. The surgical field is flooded with $\mathrm{CO}_{2}$.

Cold blood cardioplegia (Buckberg) is our preferred 
method of myocardial protection, and is given every 30 minutes.

Cerebral protection is achieved by HCA at $18-28{ }^{\circ} \mathrm{C}$ (core temperature) along with bilateral selective antegrade cerebral perfusion (SACP).

During cooling, the aortic root/ascending aortic procedure and other concomitant cardiac procedures are performed. Thereafter, the left ventricle is de-aired and the heart is perfused antegrade via a cannula inserted proximal to the aortic clamp in the ascending aorta/ascending aortic graft. Both the cardiac perfusion pressure and coronary flow are controlled (target flow: $150-200 \mathrm{~mL} /$ minute, target pressure: $60-80 \mathrm{mmHg}$ ). Flow and pressures can vary significantly depending on cardiac size and pathologies. Typically, cardiac perfusion pressures are not increased over $85 \mathrm{mmHg}$. During cardiac perfusion, the left ventricle is thoroughly vented. The aortic arch is then replaced on an empty 'beating heart'. After reaching the desired temperature, the systemic circulation is arrested and the aorta is opened. With the patient in Trendelenburg position, catheters (Medtronic DPL, USA) are introduced into the left carotid artery and the innominate artery for SACP. Cerebral perfusion is initiated at a rate of $10 \mathrm{~mL} / \mathrm{kg} / \mathrm{min}$. SACP blood temperature is kept $2{ }^{\circ} \mathrm{C}$ lower than target core temperature.

The left subclavian artery (LSA) is clamped or occluded with a Fogarty catheter (Baxter, USA).

The aorta is transected either between the left common carotid artery (LCCA) and the LSA (zone 2), or distal to the LSA (zone 3).

In patients with $\mathrm{AD}$ where the distal aortic arch is not aneurysmal, the distal anastomosis is preferably performed in zone 2.

The FET is then deployed in the proximal descending aorta. We use an endoscope to control the position of the FET after its placement in the descending aorta.

The perfusion to the lower part of the body is restarted via the fourth branch of the graft. Currently only branched aortic arch grafts are used in our center. After the distal anastomosis is completed, the proximal end of the aortic arch graft is either anastomosed to the native ascending aorta or the ascending aortic graft. In this way, the time for potential myocardial ischemia is reduced, as well as the rewarming time and consequently, the total operation time.

The LSA is then anastomosed to the third branch of the aortic arch graft and re-warming of the patient is initiated. The first and the second branch of the aortic arch graft are then anastomosed to the innominate artery and the LCCA.
Once the ECC is discontinued, the fourth branch used for antegrade perfusion is ligated and resected.

\section{Definitions}

Acute malperfusion refers to acute ischemic syndromes in patients with $\mathrm{AD}$. Chronic stroke refers to previous ischemic strokes in the patient's medical history. Permanent neurological deficits (PND) are defined as any new-onset post-operative focal neurological deficit or any focal lesion found in cerebral computed tomography (CT) or cerebral magnetic resonance tomography, irrespective of clinical symptoms. Spinal cord injury (SCI) is defined as any clinical appearance of temporary or prolonged paraplegia or paraparesis. In patients with suspected PND, SCI, or severe delirium, a neurologist was consulted. Imaging was performed according to recommendations by the neurologist.

\section{Statistical analysis}

Data were collected and analyzed retrospectively. GraphPad Prism 6 for Windows (San Diego, California, USA) was used to perform data analysis. D'Agostino \& Pearson omnibus normality test was used to analyze the distribution of data. Continuous variables were given as mean \pm standard deviation $(\mathrm{SD})$ or median + range $(\mathrm{Q} 25-\mathrm{Q} 75$, interquartile range). Categorical variables were summarized as total number $(\mathrm{N})$ and percentages.

\section{Results}

The pre-operative patient demographics are given in Table 1. Patients with degenerative aneurysms were significantly older in comparison to $\mathrm{AD}$ and $\mathrm{CD}$ patients. And, $42 \%$ of $\mathrm{AD}$ patients presented with malperfusion syndrome pre-operatively.

Peri-operative and post-operative data are given in Tables 2 and 3. Mean cardiopulmonary bypass time, aortic cross-clamp time, and myocardial ischemia time were $262 \pm 84,115 \pm 71,50 \pm 26$ minutes, respectively.

Nearly one-half of patients (43\%) underwent concomitant aortic root repair (Bentall procedure or valvesparing aortic root repair). The latter was more commonly used in $\mathrm{AD}$ patients. Operative times were significantly less in patients with degenerative aneurysms.

Overall peri-operative mortality was $12 \%$, while $17 \%$ of patients needed temporary dialysis, and only $5 \%$ of patients 


\begin{tabular}{|c|c|c|c|c|c|}
\hline Variable & All & An & $A D$ & $C D$ & $P$ value \\
\hline Number of patients & $211(100 \%)$ & $68(32 \%)$ & $96(46 \%)$ & $47(22 \%)$ & \\
\hline Operative time (min) & $395.7 \pm 109.5$ & $337.5 \pm 100.5$ & $414.7 \pm 97.6$ & $441.3 \pm 111.7$ & $<0.001$ \\
\hline Bypass time (min) & $261.5 \pm 84.4$ & $211.6 \pm 66.6$ & $290.9 \pm 84.5$ & $275.0 \pm 75.4$ & $<0.001$ \\
\hline Myocardial ischemia time (min) & $114.6 \pm 70.9$ & $93.8 \pm 58.7$ & $117.9 \pm 68.5$ & $139.4 \pm 84.0$ & 0.006 \\
\hline Lower body ischemia time (min) & $50.1 \pm 26.1$ & $41.2 \pm 22.7$ & $51.7 \pm 21.3$ & $59.3 \pm 34.6$ & $<0.001$ \\
\hline Beating heart & $184(87 \%)$ & $63(93 \%)$ & $82(85 \%)$ & $39(83 \%)$ & 0.243 \\
\hline Concomitant procedures & $112(53 \%)$ & $38(56 \%)$ & $59(61 \%)$ & $23(49 \%)$ & 0.073 \\
\hline CABG & $44(21 \%)$ & $24(35 \%)$ & $14(15 \%)$ & $6(13 \%)$ & 0.002 \\
\hline Composite root replacement & $40(19 \%)$ & $4(6 \%)$ & $23(24 \%)$ & $13(28 \%)$ & 0.003 \\
\hline David procedure & $47(22 \%)$ & $6(9 \%)$ & $35(36 \%)$ & $6(13 \%)$ & $<0.001$ \\
\hline Yacoub procedure & $3(1 \%)$ & $0(0 \%)$ & $2(2 \%)$ & $1(2 \%)$ & 0.485 \\
\hline Root stabilization & $3(1 \%)$ & $0(0 \%)$ & $3(3 \%)$ & $0(0 \%)$ & 0.162 \\
\hline Mitral valve repair & $6(3 \%)$ & $4(6 \%)$ & $1(1 \%)$ & $1(2 \%)$ & 0.175 \\
\hline Tricuspid valve surgery & $4(2 \%)$ & $2(3 \%)$ & $1(1 \%)$ & $1(2 \%)$ & 0.674 \\
\hline
\end{tabular}

$A D$, acute dissection; $C D$, chronic dissection; An, degenerative aneurysm; CABG, coronary artery bypass grafting.

\begin{tabular}{|c|c|c|c|c|c|}
\hline Variable & All & An & $A D$ & $C D$ & $P$ value \\
\hline Number of patients & $211(100 \%)$ & $68(32 \%)$ & $96(46 \%)$ & $47(22 \%)$ & \\
\hline Prolonged ventilation & $44(21 \%)$ & $10(15 \%)$ & $25(26 \%)$ & $9(19 \%)$ & 0.201 \\
\hline Rethoracotomy for bleeding & $27(13 \%)$ & $4(6 \%)$ & $15(16 \%)$ & $8(17 \%)$ & 0.113 \\
\hline Dialysis & $47(22 \%)$ & $14(21 \%)$ & $21(22 \%)$ & $12(26 \%)$ & 0.815 \\
\hline Permanent & $11(5 \%)$ & $1(1 \%)$ & $8(8 \%)$ & $2(4 \%)$ & 0.142 \\
\hline Paraparesis & $14(7 \%)$ & $4(6 \%)$ & $7(7 \%)$ & $3(4 \%)$ & 0.935 \\
\hline Permanent & $4(2 \%)$ & $3(4 \%)$ & $1(1 \%)$ & $0(0 \%)$ & 0.165 \\
\hline Stroke & $38(18 \%)$ & $9(13 \%)$ & $20(21 \%)$ & $9(19 \%)$ & 0.447 \\
\hline Delirium & $44(21 \%)$ & $16(24 \%)$ & $25(26 \%)$ & $3(4 \%)$ & 0.020 \\
\hline Recurrent nerve palsy & $40(19 \%)$ & $9(13 \%)$ & $17(18 \%)$ & $14(30 \%)$ & 0.077 \\
\hline In-hospital mortality & $25(12 \%)$ & $9(13 \%)$ & $11(11 \%)$ & $5(11 \%)$ & 0.903 \\
\hline 30-day mortality & 18 (9\%) & $5(7 \%)$ & $8(8 \%)$ & $5(11 \%)$ & 0.822 \\
\hline
\end{tabular}




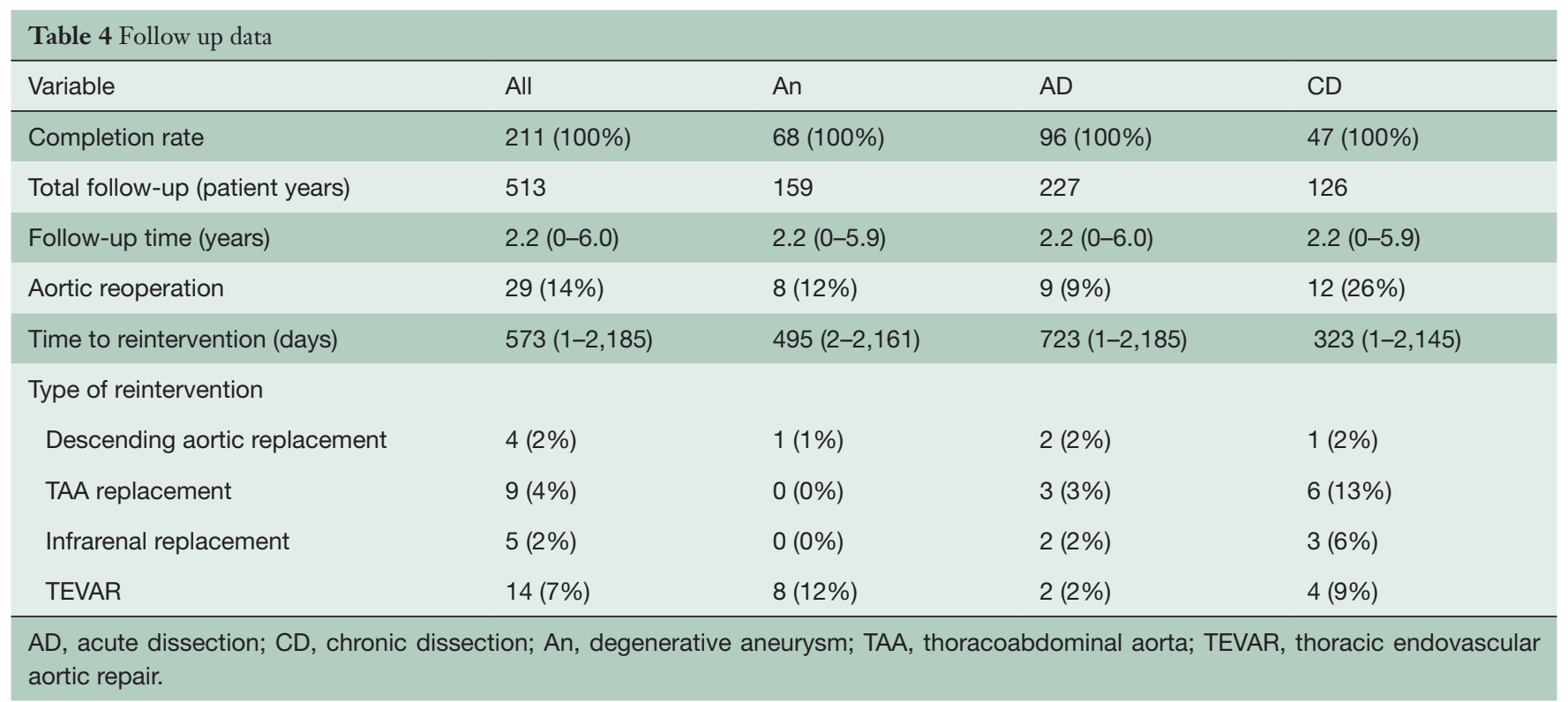

depended on dialysis at discharge. The incidence of newonset PND was $18 \%$. Temporary SCI occurred in $5 \%$ of patients, resolving with therapy until discharge. Prolonged SCI (at discharge) was found in $2 \%$ of patients.

Follow-up was complete for $100 \%$ of patients and comprised a total of 513 patient years. The mean follow-up time was 2.2 [0-6] years. During follow-up, there were 32 aortic reinterventions distal to the FET. Detailed information on distal interventions can be found in Table 4. The survival rate at 1 and 5 years was $84 \%$ and $81 \%$, respectively.

\section{Discussion}

The FET technique builds on the advantages of the classical ET technique and at the same time offers several added advantages. These advantages include the notion that complex aneurysms of the aortic arch and the proximal descending aorta can be treated in a single-stage operation (1). In case of distal aortic progression of the disease, the FET offers an ideal landing zone and may facilitate secondstage repair (3). The indications have expanded from aortic aneurysms to both acute and chronic ADs. In acute dissections with malperfusion, the FET procedure has the potential to expand and stabilize the collapsed true lumen (11).

However, the results after open aortic arch repair mainly depend on peri-operative management and patient factors. The usage of a FET - with or without a trifurcated graft-does not in itself improve the immediate peri- operative results.

Over the years, peri-operative management and operative techniques have evolved greatly. These factors have contributed towards better results after open aortic arch surgery. During the early years of FET implantation, all three supra-aortic vessels were re-implanted in the arch graft as an 'island' (en bloc technique). The introduction of the trifurcated FET graft offers the advantage of separate anastomosis of each supra-aortic vessel. This may help with more complete resection of diseased vessels in $\mathrm{AD}$ patients or dilated vessels in aneurysm patients.

\section{Aneurysm}

The FET technique offers the potential benefit of treating complex aneurysms of the aortic arch and descending aorta in a single-stage operation. However, careful patient selection, review of the pre-operative imaging, and choosing the right prosthesis to reach the distal landing zone are important in achieving this goal. If the aneurysm of the descending aorta extends beyond the level of the distal landing zone or if the disease progresses, a secondstage intervention is required. In a previous report, we have shown that the second-stage aortic completion can be facilitated by the usage of the FET (3). In the present study, one patient of the aneurysm group required open descending aortic replacement and eight patients underwent endovascular repair of the thoracic aorta. The median time 
to re-intervention was 41 months.

\section{Acute dissection}

In $\mathrm{AD}$ patients, performing a total aortic arch replacement with FET is controversial. Nevertheless, FET may be specifically helpful in the following conditions (11): first, in patients with distal malperfusion, FET implantation expands the true lumen in the descending aorta and helps in restoring visceral/lower body perfusion. Malperfusion was present in $42 \%$ of all AD patients. Following FET implantation, none of the patients required further measures in the down-stream aorta to relieve ischemia. This hypothesis has also been addressed in the position paper of the EACTS published in 2015 (11). Second, in patients with rupture of the distal aortic arch or the proximal descending aorta, FET implantation may be the only way to save the patient (11).

In the present study, $42 \%$ of $\mathrm{AD}$ patients presented with malperfusion syndrome pre-operatively. At first, this number seems to be higher than the expected incidence of malperfusion caused by acute dissection, which is approximately $30 \%$ (12). However, the higher rate in the current study can be explained by the fact that we intentionally treated patients with malperfusion with FET to re-expand the true lumen.

It is known that the mortality rate is significantly higher in $\mathrm{AD}$ patients with malperfusion (12). In light of this, the mortality rates reported in this study (30-day: $7 \%$, in-hospital: $13 \%$ ) seem to be acceptable. Other groups have reported early mortality rates ranging from $5-18 \%$ for patients with $\mathrm{AD}$ who undergo FET implantation $(10,13-15)$. Thus, the results of the current study using the trifurcated FET prosthesis seem to be comparable to the outcome of other groups.

\section{Chronic dissection}

Patients of this group include either patients with previous type B dissection or patients who underwent previous repair for type A dissection, and of whom present now with aortic arch enlargement. Careful review of the pre-operative imaging is of great importance in these patients, as reentries in the downstream aorta must be identified. This might help to prevent post-operative malperfusion caused by thrombosis of the false lumen.

In the present study, two-thirds of all CD patients had history of previous surgery, i.e., were re-operations.
Nevertheless, the in-hospital mortality rate was $11 \%$, and not significantly different when compared to elective operations for aortic aneurysm.

In order to avoid occlusion of the lumbar arteries, we have stopped using the $150 \mathrm{~mm}$ Thoraflex graft. In the present study, only $4 \%$ of CD patients had temporary paraparesis. All these patients recovered, resulting in a permanent paraparesis rate of $0 \%$. Other groups have reported SCI rates of up to $20 \%$ (6). Thus, the numbers of the current study seem to be significantly lower than the rates reported by others (6).

\section{Role of modern perioperative management techniques}

In addition to bringing the distal aortic anastomosis more proximal, it is also advisable to use other modern perioperative techniques, such as continuous myocardial perfusion (i.e., our "beating heart aortic arch surgery" technique) $(16,17)$, and spinal drainage, as described above (17), to improve overall outcome. Our data proves the importance of meticulous hemostasis to avoid bleeding, rethoracotomy, and associated adverse outcomes. We believe that the trifurcated graft allows easier hemostasis than the traditional island technique. Thus, using branched aortic arch grafts may facilitate surgical hemostasis (18). Using a combination of management changes, we were recently able to show significant improvements in outcomes after total aortic arch repair (17). The peri-operative mortality rate could be reduced from $15 \%$ to $8 \%$. Results from other groups have shown similar conclusions (19).

\section{Limitations}

This study is of retrospective nature and carries all potential disadvantages of this kind of study. To some extent, there might also be selection bias, as $\mathrm{AD}$ patients with malperfusion might have had a higher chance of receiving a FET procedure in order to re-expand the true lumen. Although the FET showed adequate outcomes in this study, the graft itself-either trifurcated or not—does not improve immediate peri-operative results.

\section{Conclusions}

Total aortic arch replacements with trifurcated FET can be performed with positive results. The trifurcated graft allows for selective anastomosis of the supra-aortic vessels, which might result in improved hemostasis. 


\section{Acknowledgments}

This study was performed with internal university funds.

\section{Footnote}

Conflicts of Interest: The authors have no conflicts of interest to declare.

Open Access Statement: This is an Open Access article distributed in accordance with the Creative Commons Attribution-NonCommercial-NoDerivs 4.0 International License (CC BY-NC-ND 4.0), which permits the noncommercial replication and distribution of the article with the strict proviso that no changes or edits are made and the original work is properly cited (including links to both the formal publication through the relevant DOI and the license). See: https://creativecommons.org/licenses/by-nc$\mathrm{nd} / 4.0 \%$.

\section{References}

1. Karck M, Chavan A, Hagl C, et al. The frozen elephant trunk technique: a new treatment for thoracic aortic aneurysms. J Thorac Cardiovasc Surg 2003;125:1550-3.

2. Borst HG, Walterbusch G, Schaps D. Extensive aortic replacement using "elephant trunk" prosthesis. Thorac Cardiovasc Surg 1983;31:37-40.

3. Rustum S, Beckmann E, Wilhelmi M, et al. Is the frozen elephant trunk procedure superior to the conventional elephant trunk procedure for completion of the second stage?. Eur J Cardiothorac Surg 2017;52:725-32.

4. Shrestha M, Pichlmaier M, Martens A, et al. Total aortic arch replacement with a novel four-branched frozen elephant trunk graft: first-in-man results. Eur J Cardiothorac Surg 2013;43:406-10.

5. Roselli EE, Idrees JJ, Bakaeen FG, et al. Evolution of Simplified Frozen Elephant Trunk Repair for Acute DeBakey Type I Dissection: Midterm Outcomes. Ann Thorac Surg 2018;105:749-55.

6. Kfuri M, Schatzker J. Response to A. Kumar, et al., Letter to the Editor concerning "Revisiting the Schatzker classification of tibial plateau fractures" by Kfuri $M$, Schatzker J. Injury 201849 December (12):2252-2263, Injury (2019) https://doi.org/10.1016/j.Injury.2019.01.020. Injury 2019;50:1262-4.

7. Shrestha M, Kaufeld T, Beckmann E, et al. Total aortic arch replacement with a novel 4-branched frozen elephant trunk prosthesis: Single-center results of the first 100 patients. J Thorac Cardiovasc Surg 2016;152:148-59.e1.

8. Leone A, Di Marco L, Coppola G, et al. Open distal anastomosis in the frozen elephant trunk technique: initial experiences and preliminary results of arch zone 2 versus arch zone 3†. Eur J Cardiothorac Surg 2019;56:564-71.

9. Jakob H, Dohle D, Benedik J, et al. Long-term experience with the E-vita Open hybrid graft in complex thoracic aortic disease†. Eur J Cardiothorac Surg 2017;51:329-38.

10. Chen X, Huang F, Xu M, et al. The stented elephant trunk procedure combined total arch replacement for Debakey I aortic dissection: operative result and follow-up. Interact Cardiovasc Thorac Surg 2010;11:594-8.

11. Shrestha M, Bachet J, Bavaria J, et al. Current status and recommendations for use of the frozen elephant trunk technique: a position paper by the Vascular Domain of EACTS. Eur J Cardiothorac Surg 2015;47:759-69.

12. Erbel R, Aboyans V, Boileau C, et al. 2014 ESC Guidelines on the diagnosis and treatment of aortic diseases: Document covering acute and chronic aortic diseases of the thoracic and abdominal aorta of the adult. The Task Force for the Diagnosis and Treatment of Aortic Diseases of the European Society of Cardiology (ESC). Eur Heart J 2014;35:2873-926.

13. Pochettino A, Brinkman WT, Moeller P, et al. Antegrade thoracic stent grafting during repair of acute DeBakey I dissection prevents development of thoracoabdominal aortic aneurysms. Ann Thorac Surg 2009;88:482-9; discussion 489-90.

14. Uchida N, Katayama A, Tamura K, et al. Frozen elephant trunk technique and partial remodeling for acute type A aortic dissection. Eur J Cardiothorac Surg 2011;40:1066-71.

15. Xiao Z, Meng W, Zhu D, et al. Treatment strategies for left subclavian artery during total arch replacement combined with stented elephant trunk implantation. J Thorac Cardiovasc Surg 2014;147:639-43.

16. Martens A, Koigeldiyev N, Beckmann E, et al. Do not leave the heart arrested. Non-cardioplegic continuous myocardial perfusion during complex aortic arch repair improves cardiac outcome. Eur J Cardiothorac Surg 2016;49:141-8.

17. Martens A, Beckmann E, Kaufeld T, et al. Total aortic arch repair: risk factor analysis and follow-up in 199 patients. Eur J Cardiothorac Surg 2016;50:940-8.

18. Shrestha M, Martens A, Behrendt S, et al. Is the branched 
graft technique better than the en bloc technique for total aortic arch replacement?. Eur J Cardiothorac Surg 2014;45:181-6; discussion 186-7.

19. Tsagakis K, Dohle D, Benedik J, et al. Overall Essen's

Cite this article as: Beckmann E, Martens A, Korte W, Kaufeld T, Krueger H, Haverich A, Shrestha M. Open total arch replacement with trifurcated graft and frozen elephant trunk. Ann Cardiothorac Surg 2020;9(3):170-177. doi: 10.21037/acs.2020.03.09 experience with the E-vita open hybrid stent graft system and evolution of the surgical technique. Ann Cardiothorac Surg 2013;2:612-20. 